\title{
COMMENT
}

Check for updates

\section{COVID-19 vaccination in kidney transplant recipients}

Sophie Caillard $\mathbb{1}^{1} \bowtie$ and Olivier Thaunat $\mathbb{1}^{2 凶}$

Kidney transplant recipients receive therapeutic immunosuppression that impairs their immune responses to the COVID-19 mRNA vaccine. For this reason, this vulnerable patient population is insufficiently protected by the standard two-dose COVID-19 vaccination programme and requires a specific follow-up to guide personalization of an intensified vaccination approach.

only $4-48 \%$ of kidney transplant recipients have detectable anti-spike IgGs after receiving two vaccine doses

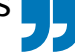

${ }^{1}$ Department of Nephrology and Transplantation, University Hospitals of Strasbourg, INSERM Unit 1109, Strasbourg, France.

\section{${ }^{2}$ Department of}

Transplantation Nephrology and Clinical Immunology Hospices Civils de Lyon, Claude Bernard Lyon I University, INSERM Unit 1111, Lyon, France.

凶e-mail: sophie.caillard@ chru-strasbourg.fr; olivier. thaunat@chu-lyon.fr

https://doi.org/10.1038/ s41581-021-00491-7
Patients with kidney disease are at high risk of developing severe COVID-19. This risk is especially high in kidney transplant recipients, in whom the risk of death owing to SARS-CoV-2 infection is estimated to be as high as $25 \%$ (REF. ${ }^{1}$ ). Consequently, the health authorities of numerous countries have prioritized this vulnerable population for vaccination with $m R N A$ vaccines.

The BNT162b2 (Pfizer/BioNTech) and mRNA-1273 (Moderna) vaccines comprise lipid nanoparticles containing nucleoside-modified mRNA molecules that encode the SARS-CoV-2 spike protein. The use of antigen-encoding RNA for vaccination is a fast, highly scalable, innovative technology that is also pharmaceutically safe because the RNA is transiently expressed and the risk of integration into the genome of the treated host is low ${ }^{2}$. Moreover, in addition to inducing potent $\mathrm{CD} 4{ }^{+}$helper $\mathrm{T}$ cell and antibody responses, which are also induced by protein-based immunizations, mRNA vaccines in general (and BNT162b2 and mRNA-1273 in particular) induce strong $\mathrm{CD}^{+}$cytotoxic $\mathrm{T}$ cell responses that can eliminate infected cells, which function as 'virus factories' ${ }^{2,3}$.

Whether protection against COVID-19 after mRNA vaccination depends mainly on cellular (cytotoxic cells) or humoral (antibodies) adaptive immune effectors termed mechanistic correlates of protection - is not yet entirely clear and might vary depending on factors such as the virus variant or patient characteristics. However, recent reports have clearly demonstrated the importance of developing neutralizing antibodies ${ }^{4}$, in particular in vaccinated kidney transplant recipients ${ }^{5}$. Neutralizing antibodies act by binding to the spike protein of SARS-CoV-2, thereby eliminating extracellular viruses and preventing their entry into host cells. Importantly, high titres of anti-spike IgGs (>264 binding antibody units per $\mathrm{ml}$ ), which can be measured by commercial serological tests and are much easier to perform at large scale than neutralization assays, correlate well with the presence of neutralizing antibodies and vaccine efficacy against primary symptomatic COVID-19.
Although in the general population both BNT162b2 and mRNA-1273 vaccines induce robust titres of antispike IgGs that confer $>94 \%$ protection against severe COVID-19, many independent studies (Supplementary Table 1) have reported that only $4-48 \%$ of kidney transplant recipients have detectable anti-spike IgGs after receiving two vaccine doses. Consequently, severe cases of COVID-19 still occur in vaccinated transplant recipients ${ }^{6}$, which demonstrates that they are insufficiently protected by the current 'standard' COVID-19 vaccination approach. Kidney transplant recipients who have not been previously infected with SARS-CoV-2 are more likely to generate anti-spike IgGs if vaccination is performed late after transplantation (probably owing to lower levels of immunosuppression), if they have a high glomerular filtration rate and if circulating anti-spike IgGs are detectable after the first dose of vaccine. Conversely, older age, diabetes and high levels of maintenance immunosuppression are all associated with a lower vaccination response rate. Some evidence indicates that patients treated with belatacept (a T cell co-stimulation blocker) or rituximab (used for B cell depletion) display low $(<10 \%)$ seroconversion rates (Supplementary Table 1).

A suboptimal response to COVID-19 mRNA vaccines in transplant recipients was not entirely unexpected because these patients are known to mount poor responses to many protein subunit vaccines. Maintenance immunosuppression regimens used to prevent graft rejection commonly include a calcineurin inhibitor, which interferes with $\mathrm{T}$ cell activation, and an antimetabolite, such as mycophenolate mofetil (MMF), which blocks the proliferation of activated $\mathrm{T}$ and $\mathrm{B}$ cells. Although these drugs, particularly when used in combination and/or at high doses, impede antibody generation after vaccination, several reports suggest that MMF, which blocks the proliferation of follicular helper $\mathrm{CD}^{+} \mathrm{T}$ $\left(\mathrm{T}_{\mathrm{FH}}\right.$ cells), is especially detrimental. In our preprint study, $\mathrm{T}_{\mathrm{FH}}$ cells were instrumental for the differentiation of spike-specific B cells into antibody-producing plasma cells ${ }^{5}$. 


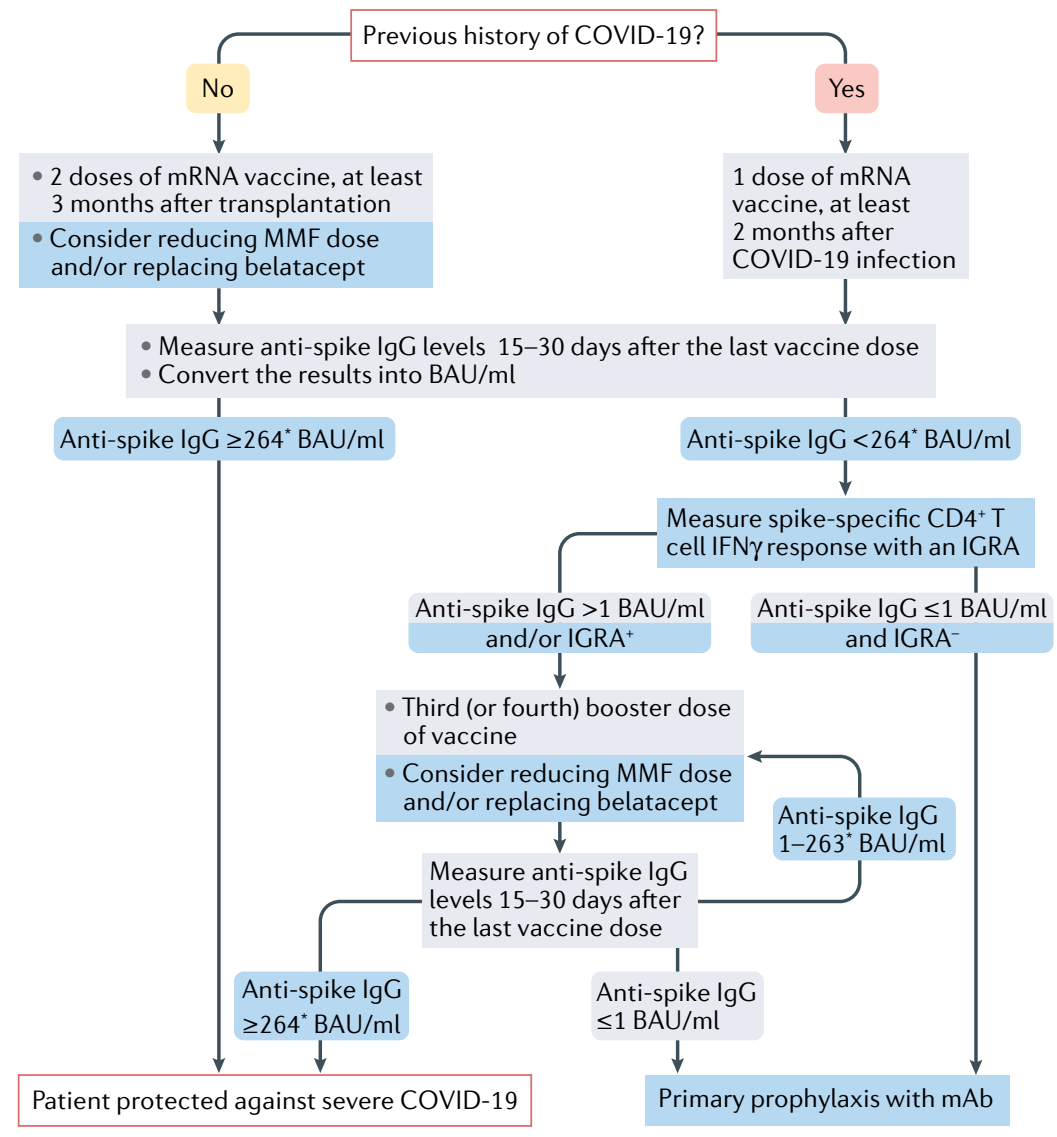

Based on concordant independent studies

Based on recent preliminary results that require further validation

Fig. 1 | Suggested vaccination approach for protection against COVID-19 in kidney transplant recipients. The presence of SARS-CoV-2-neutralizing antibodies and vaccine efficacy against primary symptomatic COVID-19 correlates well with anti-spike protein $\lg G$ titres, which can be measured with commercial serological tests ${ }^{4}$. Another advantage of these antigen-binding assays is that their results can be converted to the WHO international standard (NIBSC code 20/136) and expressed in binding antibody units (BAU)/ml, making individual results comparable across different platforms and laboratories. Most reports suggest that the antibody response peaks 15 days after the last dose of vaccine and remains stable until at least day 30 . Follicular helper $C D 4^{+} T\left(T_{F H}\right)$ cells seem to be instrumental for the differentiation of spike-specific $B$ cells into antibody-producing plasma cells. However, enumeration of spike-specific $\mathrm{T}_{\mathrm{FH}}$ cells in the circulation of patients requires complex techniques that are not available in routine clinical practice. We have demonstrated in a preprint study that IFN $\gamma$ levels measured with commercially available IFN $\gamma$ release assays (IGRAs) performed within 30 days after the last dose of vaccine correlate with the number of spike-specific $\mathrm{T}_{\mathrm{FH}}$ cells ${ }^{8}$. $\mathrm{mAb}$, monoclonal antibodies; MMF, mycophenolate mofetil. *This threshold was defined in one preprint publication only and may vary according to the virus variants and the neutralization tests used.

increasing vaccine immunogenicity might be a safer approach to improve vaccine efficacy than reducing immunosuppression

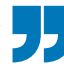

Approaches to improve COVID-19 vaccine efficacy in transplant recipients include modulation of immunosuppression - for example, temporary reduction or suspension of MMF treatment, or belatacept replacement - and should be evaluated on an individual basis owing to the potential risk of graft rejection. Importantly, the immune mechanisms that enable the generation of protective anti-spike IgGs after vaccination also underlie the generation of anti-donor HLA antibodies, which are widely recognized as the main cause of late kidney allograft rejection. Notably, infection with SARS-CoV-2 usually triggers the generation of high titres of protective neutralizing anti-spike IgGs in kidney transplant recipients ${ }^{5}$, and the main predictor of successful seroconversion after vaccination in these patients is a previous history of COVID-19; in these patients, a single dose of mRNA vaccine leads to a $100 \%$ seroconversion rate and antibody levels that are comparable to those observed in healthy volunteers?. These observations suggest that increasing vaccine immunogenicity might be a safer approach to improve vaccine efficacy than reducing immunosuppression.

Strategies to optimize protein subunit vaccine immunogenicity in kidney transplant recipients include the use of adjuvants, intradermal injection and high antigen doses, but these approaches have not yet been tested for COVID-19 mRNA vaccines. Given that the post-influenza vaccine seroconversion rate in kidney transplant recipients improved when additional 'booster' doses were received, administration of a third dose of COVID-19 mRNA vaccine to these patients has been authorized in France (April 2021) and in the USA (August 2021). Although a third vaccine dose was globally very well tolerated by kidney transplant recipients who had an insufficient anti-spike IgG response after two vaccine doses, the serological response was heterogeneous (Supplementary Table 1). Overall, this intensified vaccination approach led to a protective titre of anti-spike IgG in $\sim 40 \%$ of kidney transplant recipients. In a preprint study, predictive variables associated with a successful response to the third vaccine dose included the presence of anti-spike IgGs and/or spike-specific IFN $\gamma$-producing $\mathrm{CD}^{+}{ }^{+} \mathrm{T}$ cells after the second dose $e^{8}$. By contrast, $<10 \%$ of kidney transplant recipients with negative serology and a negative IFN $\gamma$ releasing assay responded to the third $\operatorname{dose}^{8}$. These patients might instead benefit from passive transfer of anti-SARS-CoV-2 monoclonal antibodies.

Given their increased vulnerability to severe COVID-19, kidney transplant recipients need to be effectively vaccinated. Although kidney transplantation candidates receiving chronic haemodialysis also have a defective response to the COVID-19 mRNA vaccine ${ }^{9}$, this defect is milder than that observed in transplant recipients being treated with immunosuppressive drugs. COVID-19 vaccination should therefore be administered during the pre-transplant waiting period. Transplant recipients with a previous history of COVID-19 usually respond well to a single dose of mRNA vaccine but, for those without prior infection, assessment of anti-spike IgG titres after the second dose of mRNA vaccine might be important to discriminate patients who are adequately protected from those likely to lack neutralizing antibodies ${ }^{10}$. In SARS-CoV-2-naive kidney transplant recipients, determining anti-spike IgG levels after two doses of vaccine (and, when possible, an estimation of the number of spike-specific $\mathrm{CD} 4^{+} \mathrm{T}$ cells using a simple commercially available IFN $\gamma$ release assay) might be useful to guide the administration of a third or fourth booster dose, or the use of primary prophylaxis with monoclonal antibodies (FIG. 1). Finally, although accumulating data generated all over the world during the first part of 2021 have enabled the development of a vaccination approach against COVID-19, data regarding the duration of protection 
(that is, the stability of neutralizing antibody titres over time) are lacking, and the crucial question of whether kidney transplant recipients will need boosters (and when) remains to be investigated.

1. Caillard, S. et al. An initial report from the French SOT COVID Registry suggests high mortality due to COVID-19 in recipients of kidney transplants. Kidney Int. 98, 1549-1558 (2020).

2. Pardi, N., Hogan, M. J., Porter, F. W. \& Weissman, D. mRNA vaccines - a new era in vaccinology. Nat. Rev. Drug Discov. 17, 261-279 (2018)

3. Sahin, U. et al. BNT162b2 vaccine induces neutralizing antibodies and poly-specific T cells in humans. Nature. 595, 572-577 (2021).

4. Khoury, D. S. et al. Neutralizing antibody levels are highly predictive of immune protection from symptomatic SARS-CoV-2 infection. Nat. Med. 27, 1205-1211 (2021).

5. Charmetant, X. et al. Comparison of infected and vaccinated transplant recipients highlights the role of Tfh and neutralizing IgG in COVID-19 protection. Preprint at medRxiv https://doi.org/ 10.1101/2021.07.22.21260852 (2021).
6. Caillard, S. et al. Occurrence of severe COVID-19 in vaccinated transplant patients. Kidney Int. 100, 477-479 (2021).

7. Benotmane, I. et al. Strong antibody response after a first dose of a SARS-CoV-2 mRNA-based vaccine in kidney transplant recipients with a previous history of COVID-19. Am. J. Transplant. https://doi.org/10.1111/ajt.16764 (2021).

8. Charmetant, X. et al. Predictive factors of response to 3rd dose of COVID-19 mRNA vaccine in kidney transplant recipients. Preprint at medRxiv https://doi.org/10.1101/2021.08.23.21262293 (2021).

9. Espi, M. et al. The ROMANOV study found impaired humoral and cellular immune responses to SARS-Cov-2 mRNA vaccine in virus unexposed patients receiving maintenance hemodialysis. Kidney Int 100, 928-936 (2021).

10. Feng, S. et al. Correlates of protection against symptomatic and asymptomatic SARS-CoV-2 infection. Preprint at medRxiv https://doi.org/10.1101/2021.06.21.21258528 (2021).

\section{Competing interests}

The authors declare no competing interests.

Supplementary information

Supplementary information is available for this paper at https://doi.org/ 10.1038/s41581-021-00491-7. 